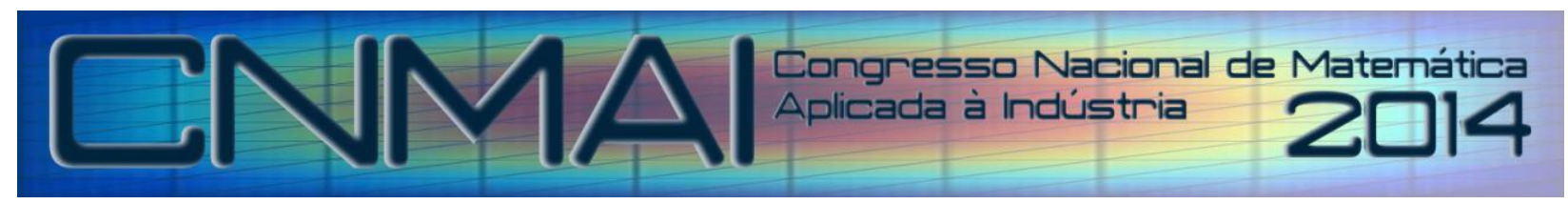

18 a 21 de novembro de 2014, Caldas Novas - Goiás

\title{
MODELAGEM MATEMÁTICA DA TRANSMISSÃO MECÂNICA EM UMA JUNTA ROBÓTICA COM FUSO DE ESFERAS
}

\author{
Angelo Fernando Fiori, an@ unochapeco.edu.br ${ }^{1}$ \\ Sandra Baratto Viecelli, sandra_edinara@hotmail.com² \\ Leonardo Bortolon Maraschin, leonardo.maraschin@unijui.edu.br ${ }^{3}$ \\ Ismael Barbieri Garlet, Ismael.garlet@ hotmail.com ${ }^{4}$ \\ Antonio Carlos Valdiero, valdiero@unijui.edu.br ${ }^{5}$ \\ Luiz Antonio Rasia, rasia@unijui.edu.br ${ }^{6}$
}

${ }^{1}$ Universidade Regional do Noroeste do Estado do Rio Grande do Sul - Departamento de Ciências Exatas e Engenharias (DCEEng) ... Rua Prefeito Rudi Franke, 540, Arco Íris - 98280000, Panambi (RS)

${ }^{2}$ Universidade Regional do Noroeste do Estado do Rio Grande do Sul - Departamento de Ciências Exatas e Engenharias (DCEEng) ... Rua Prefeito Rudi Franke, 540, Arco Íris - 98280000, Panambi (RS)

${ }^{3}$ Universidade Regional do Noroeste do Estado do Rio Grande do Sul - Departamento de Ciências Exatas e Engenharias (DCEEng) ... Rua Prefeito Rudi Franke, 540, Arco Íris - 98280000, Panambi (RS)

${ }^{4}$ Universidade Regional do Noroeste do Estado do Rio Grande do Sul - Departamento de Ciências Exatas e Engenharias (DCEEng) ... Rua Prefeito Rudi Franke, 540, Arco Íris - 98280000, Panambi (RS)

${ }^{5}$ Universidade Regional do Noroeste do Estado do Rio Grande do Sul - Departamento de Ciências Exatas e Engenharias (DCEEng) ... Rua Prefeito Rudi Franke, 540, Arco Íris - 98280000, Panambi (RS)

${ }^{6}$ Universidade Regional do Noroeste do Estado do Rio Grande do Sul - Departamento de Ciências Exatas e Engenharias (DCEEng) ... Rua Prefeito Rudi Franke, 540, Arco Íris - 98280000, Panambi (RS)

Resumo: Tem-se por objetivo desenvolver a modelagem matemática das características não lineares da transmissão mecânica em um robô industrial do tipo Gantry e apresentar o projeto de um protótipo. O robô é constituído de juntas prismáticas de fuso de esferas e acionado por motores elétricos de corrente alternada. O torque gerado na saída do motorredutor resulta no deslocamento angular do eixo do fuso, cujo movimento é transformado no deslocamento linear de um elo em relação ao anterior em direções ortogonais, fazendo-o transladar. A formulação matemática das equações da posição nas juntas prismáticas torna-se uma tarefa mais fácil devido ao desacoplamento cinemático entre as juntas ortogonais do robô. Os dados de posição e torque podem ser obtidos a partir de experimentos, onde os sinais de sensores são capturados através de uma placa de aquisição e controle (dSPACE). No entanto, as transmissões mecânicas possuem não linearidades que dificultam o controle de precisão e prejudicam o desempenho, acarretando atrasos e perdas de movimento. Como a modelagem se dará no sistema de transmissão motorredutor-acoplamentofuso-castanha, tem-se a formulação dos modelos matemáticos através do método Newton-Euler no equilíbrio dinâmico a partir dos diagramas de corpo livre do eixo-fuso e da porca-massa deslocada. Obtêm-se, assim, duas equações diferenciais ordinárias de segunda ordem (uma para a rotação e outra para a translação). Devido a existência da não linearidade da folga, essas duas equações são combinadas e acopladas através de uma relação matemática em tempo discretizado. Este modelo dinâmico de junta prismática é generalizado para os dois graus de liberdade. Como perspectivas futuras do trabalho, prevê-se a validação experimental no protótipo do robô já em fase de construção. Espera-se que este trabalho contribua em esquemas de compensação das não linearidades e do controle preciso de robôs industriais de baixo custo em tarefas insalubres, perigosas e de grande esforço mecânico.

Palavras-chave: Modelagem Matemática, Fuso de Esferas, Não linearidade de Folga.

\section{INTRODUÇÃO}


O presente trabalho trata da apresentação do projeto do protótipo de um robô Gantry e da descrição da folga enquanto características não linear presente nas transmissões do tipo fuso de esferas, além de utilizar a convenção de Denavit-Hartenberg para a modelagem cinemática direta da descrição de relações entre um elo e outro.

Robôs Gantry são amplamente utilizados na indústria em funções de guincho robô, máquinas de corte laser e usinagem CNC por apresentarem vantagens na produtividade, qualidade, segurança e adaptabilidade (Shang e Cong, 2014) além de serem robustos (Paatz, 2008), permitindo o deslocamento de grandes cargas. Robôs deste tipo são compostos por juntas prismáticas, com eixos perpendiculares acarretando no desacoplamento cinemático, o que facilita a programação e modelagem, sendo decisivo nos efeitos de acionamento. Tais efeitos são muito importantes para a modelagem dinâmica, haja vista que afetam características dinâmicas do sistema de alimentação da máquina-ferramenta e, portanto, afetam a qualidade final da operação (Zhang et al, 2013).

Assim, a escolha da transmissão deve levar em consideração a potência necessária e o tipo de movimento a ser desempenhado, optando-se por correias, cabos, engrenagens e eixo-fuso de esferas, por exemplo, ou pela combinação de sistemas de transmissão. O deslocamento da massa, neste caso, se dá através de um fuso de esferas o qual, segundo Valdiero (2005), possui alta eficiência, baixo atrito, média rigidez e alta precisão ao mesmo tempo em que possui alto atrito estático o que pode resultar na variação cíclica do torque, mas exibe a não linearidade da folga como grande desvantagem. A utilização de sensores aumenta, segundo Douat et al (2014) a precisão de robôs paralelos com dois graus de liberdade pela possibilidade de reprogramar a operação e compensar tal não linearidade.

Outro fator decisivo é a escolha do acionamento. Eles devem contemplar a necessidade do projeto (adequado a cada utilidade do robô), custos iniciais e de manutenção. A indústria, de modo geral, utiliza-se de três tipos de acionamento e de suas combinações: pneumáticos, hidráulicos e elétricos. Sistemas pneumáticos e hidráulicos exibem vantagens especialmente relacionadas à boa relação peso/potência e a partidas e paradas rápidas além de, no caso da pneumática, utilizar-se de um fluído de potência limpo (ar). As desvantagens estão especialmente relacionadas ao alto custo, perdas de potência por vazamentos e/ou dissipação por atrito viscoso além de elevada dependência da temperatura (Von Linsingen, 2003). Já nos sistemas acionados por motores elétricos (CA ou CC) as vantagens são invariância da velocidade, operação degradada em baixa carga (baixos rendimentos e fator de potência), alta corrente de partida, eficiência do processo, tempos de ciclo mais curtos (resposta dinâmica melhor) e menor manutenção tendo como principais desvantagens o elevado custo e partidas e paradas lentas (Garcia, 2003).

Nas mais diversas aplicações do manipulador robótico do tipo Gantry na indústria, é sempre muito importante saber a posição do efetuador final, bem como de suas juntas, especialmente pelo cálculo do centro de gravidade e da atividade do efetuador final dentro de um espaço de trabalho. Assim surge a necessidade de modelar matematicamente o robô para o controle de posição.

A modelagem cinemática direta em robôs é feita utilizando-se a convenção de Denavit-Hartenberg (D-H), descrevendo os elos e as relações entre eles (Sciavicco E Siciliano, 1996). A notação D-H sistematiza a descrição da cinemática de posição dos sistemas mecânicos articulados com $n$ graus de liberdade (Romano, 2002) através de uma notação sistemática a qual atribui a cada elo um sistema ortonormal (Schilling, 1990). A notação D-H baseia-se em apenas dois parâmetros, haja vista que para se determinar a posição relativa de duas retas no espaço são necessários tão somente dois parâmetros também. A partir dos parâmetros encontrados nas relações dos sistemas de coordenadas, se pode calcular as matrizes de transformação homogênea, que relacionam o movimento de um elo em relação ao elo anterior bem como a matriz de transformação homogênea, que relaciona o sistema de referência do efetuador final com o sistema de referência da base fixa (Nof, 1999).

A grande possibilidade de combinar acionamentos e transmissões, aliados ao processo contínuo de melhoria no controle de sistemas robóticos, que aprimoraram a funcionalidade e adaptabilidade de robôs do tipo Gantry, conduziram, segundo Po-Nagen (2009), ao aumento da complexidade e elevação de preços dos sistemas robóticos. Estes dois fatos fazem com que as pesquisas, especialmente na engenharia e na matemática aplicada, auxiliem no desenvolvimento de estratégias mais baratas e menos complexas sem perder a qualidade na execução do processo.

Este trabalho utiliza-se de pesquisa bibliográfica (descritiva e exploratória) para atingir seu objetivo de apresentar o projeto do protótipo desenvolvido e a modelagem matemática da cinemática direta e da não linearidade da folga, como contribuição para a robotização de baixo custo e o desenvolvimento de estratégias futuras para compensação da folga e o controle de precisão. Para isso, este trabalho está organizado em três seções. Na primeira é apresentado o projeto do robô Gantry, na seção seguinte a modelagem matemática da não linearidade da folga e na terceira a modelagem matemática da cinemática direta do robô. Ao final são apresentadas as conclusões e perspectivas de trabalhos futuros bem como os agradecimentos.

\section{PROJETO DO ROBÔ GANTRY}

O robô Gantry é caracterizado pela sua estrutura cinemática cartesiana, onde o primeiro elo é uma base fixa na forma de um pórtico, e os demais elos são unidos por juntas prismáticas, permitindo o posicionamento do efetuador final (garra ou ferramenta), conforme Fig. 1. Os movimentos de translação são descritos em planos cartesianos de três dimensões que dão o espaço de trabalho do robô. 


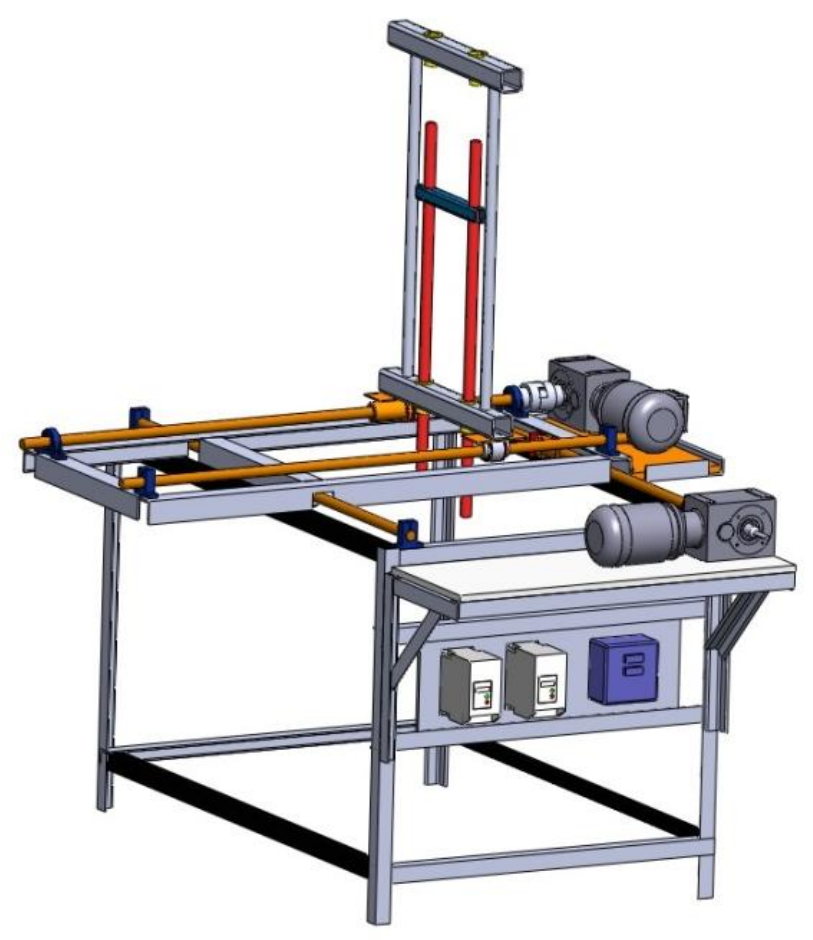

Figura 1 - Robô tipo Gantry com transmissão por fuso de esferas e acionamento elétrico

No manipulador robótico, o motorredutor de corrente alternada é montado em uma das extremidades do fuso, aplicando neste um torque que resulta em um deslocamento angular no fuso o que por sua vez provoca um deslocamento linear na carga.

A ligação entre a barra guia e o motorredutor se dá através de acoplamento elástico que permite desalinhamentos. O controle dos movimentos será executado através de uma placa eletrônica ligada a dois conversores de frequência, onde a partir da qual, os sinais serão recebidos e enviados através de uma placa de aquisição e controle dSPACE DS 1102 por comandos do Simulink/MatLab ${ }^{\circledR}$. Em cada ponta do fuso são instalados encoders incrementais. Além dos encoders, são utilizados transdutores de deslocamento sem contato (Balluff) para medir os deslocamentos. Na Fig. 2 estão indicados os elementos constitutivos do robô.



Figura 2 - Componentes do protótipo do robô Gantry com acionamento elétrico 
Os componentes indicados na Fig. 2 estão descritos na Tab. 1 com seus fabricantes e principais características técnicas e farão parte do protótipo. Para a construção foram seguidas as especificações técnicas dos fabricantes e a normativas vigentes de modo a, posteriormente, simular e validar os modelos em comportamentos do robô a partir de comando enviados através do computador.

Tabela 1 - Especificações técnicas dos componentes do protótipo

\begin{tabular}{|c|c|c|c|}
\hline Esquema & Item & Fabricante & Características Importantes \\
\hline 1 & $\begin{array}{l}\text { Guia linear tipo } \\
\text { fuso }\end{array}$ & Kalatec & $\begin{array}{l}\text { Diâmetro: } 25 \mathrm{~mm} \text {; Comprimento } 1265 \mathrm{~mm} \text {; Capacidade carga } \\
\text { dinâmica: } 100 \mathrm{~kg} \text {; Capacidade de carga estática } 159 \mathrm{~kg} \text {; }\end{array}$ \\
\hline 2 & $\begin{array}{l}\text { Guia linear tipo } \\
\text { lisa }\end{array}$ & Kalatec & $\begin{array}{l}\text { Diâmetro: } 25 \mathrm{~mm} \text {; Comprimento } 1200 \mathrm{~mm} \text {; Capacidade carga } \\
\text { dinâmica: } 100 \mathrm{~kg} \text {; Capacidade de carga estática } 159 \mathrm{~kg} ;\end{array}$ \\
\hline 3 & $\begin{array}{l}\text { Fuso de esferas } \\
\text { métrico }\end{array}$ & Kalatec & $\begin{array}{l}\text { Diâmetro 25mm; Passo: } 10 \mathrm{~mm} \text {; Folga axial: } 0,025 \mathrm{~mm} \text {; } \\
\text { Capacidade carga dinâmica: } 2954 \mathrm{~kg} \text {; Capacidade carga estática } \\
\text { 7295kg; Desvio do passo: } 0,05 \mathrm{~mm} \text {; Classe de precisão: } 300 \mathrm{~mm} \text {; }\end{array}$ \\
\hline 4 & $\begin{array}{l}\text { Anel de retenção } \\
\text { de eixo }\end{array}$ & Reno & Diâmetro: 25mm; Capacidade: $3340 \mathrm{Kg} / \mathrm{mm}$; \\
\hline 5 & $\begin{array}{l}\text { Acoplamento } \\
\text { elástico }\end{array}$ & Acriflex & Desalinhamento angular de 0,0175 rad e radial de $2 \mathrm{~mm}$; \\
\hline 6 & Mancal liso & Kalatec & $\begin{array}{l}\text { Diâmetro: } 25 \text { mm; Capacidade carga dinâmica: } 100 \text { kg; } \\
\text { Capacidade de carga estática } 159 \mathrm{~kg} ;\end{array}$ \\
\hline 7 & $\begin{array}{l}\text { Mancal com } \\
\text { rolamento }\end{array}$ & Kalatec & $\begin{array}{l}\text { Diâmetro: } 25 \text { mm; Capacidade carga dinâmica: } 100 \mathrm{~kg} \text {; } \\
\text { Capacidade de carga estática 159kg; }\end{array}$ \\
\hline 8 & Motor trifásico & Nova & Potência: $750 \mathrm{~W} / 220 \mathrm{~V} ; 1730 \mathrm{rpm}$, Torque: $12,7486 \mathrm{Nm}$; \\
\hline 9 & Motorredutor & ATI Brasil & Potência: $750 \mathrm{~W} ; 85$ rpm de rotação de saída; \\
\hline 10 & Encoder & Hohner & Passo: 5mm; 1000 pulsos por rotação; \\
\hline
\end{tabular}

Nas mais diversas aplicações do manipulador robótico do tipo Gantry, bem como em todos os robôs, é sempre muito importante saber a posição do efetuador final, bem como de suas juntas, especialmente pelo cálculo do centro de gravidade e da atividade do efetuador final dentro de um espaço de trabalho. Assim surge a necessidade de modelar matematicamente o robô para o controle de posição.

\section{MODELAGEM MATEMÁTICA DA NÃO LINEARIDADE DA FOLGA}

O problema proposto é a modelagem de um robô Gantry com transmissão mecânica tipo fuso de esferas e acionamento por motor elétrico trifásico tipo motorredutor de corrente alternada considerando-se como entrada do sistema o torque motor $T_{m}$. Sendo assim, os modelos que descrevem as dinâmicas da junta prismática para a folga são além de não lineares, dinâmicos e determinísticos, para os quais serão adotadas como hipóteses simplificadoras as seguintes condições:

- A folga (backlash) é constante em toda a extensão do fuso, não sendo considerada a folga devido ao uso do sistema;

- O atrito é viscoso e ocorre durante os deslocamentos angulares do eixo e também dos movimentos lineares da massa deslocada;

- Não será considerado o atrito dinâmico;

- Os elementos da transmissão são corpos rígidos (se despreza a elasticidade).

Como a modelagem se dará no sistema de transmissão motorredutor-acoplamento-fuso-castanha, tem-se a formulação dos modelos matemáticos da junta prismática por fuso através do método Newton-Euler, como descrito em Sciavicco e Siciliano (1996), a partir do equilíbrio dinâmico no diagrama de corpo livre do eixo-fuso e da porca-massa deslocada.

A junta prismática do tipo fuso transforma rotações em translações. O modelo para tal junta em um robô Gantry pode ser obtido através do método de Newton-Euler (onde cada corpo rígido é considerado separadamente, a representação cinemática é dada por matrizes de rotação e vetores de posição e as equações de movimento são recursivas) conforme a Fig. 3 a seguir. 


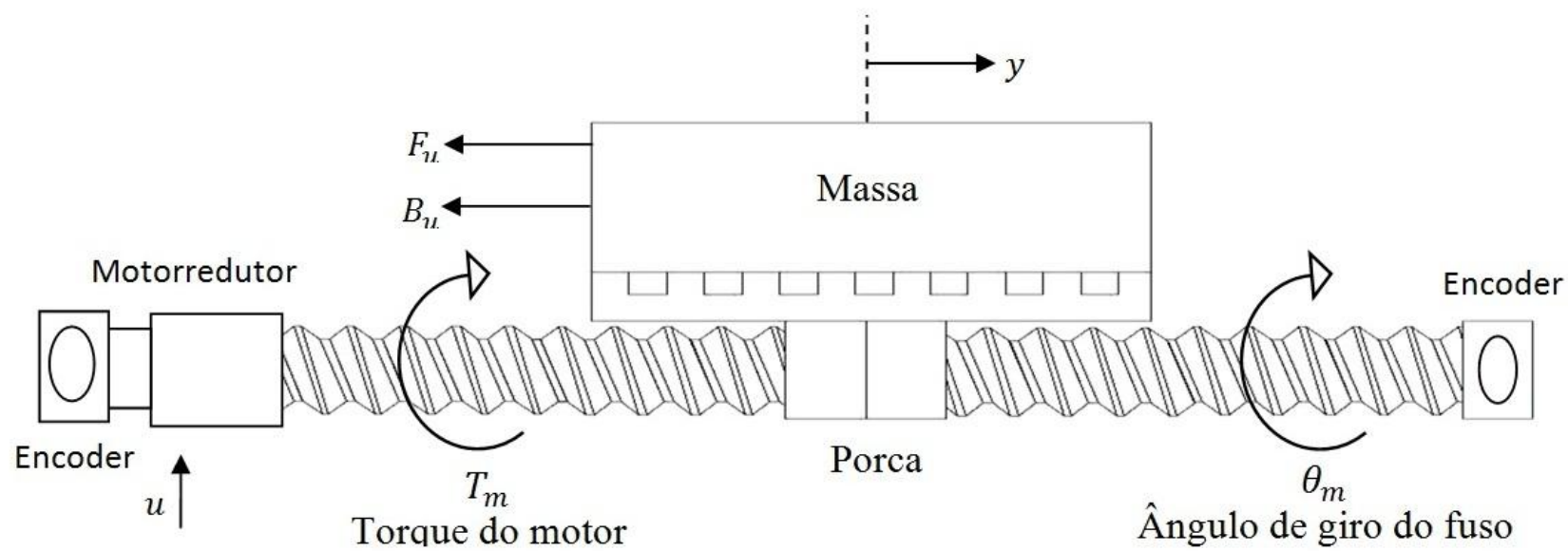

Figura 3 - Forças atuantes na dinâmica da porca

Pela lei do equilíbrio dinâmico, tem-se:

$$
\sum T=J \ddot{\theta}_{m}
$$

De acordo com a Fig. 3, tem-se que um torque do motor $\left(T_{m}\right)$ aplicado no fuso e dois torques de resistência contrários ao $T_{m}$ (torque de atrito e o torque devido a força de reação d/a massa deslocada sobre o fuso) produzem um deslocamento angular $\theta_{m}$ resultante (ângulo de giro do fuso), conforme descrito na Eq. (1). Logo,

$$
T_{m}-B_{\theta} \dot{\theta}_{m}+\frac{F_{u} p}{2 \pi}=J \ddot{\theta}_{m}
$$

Reescrevendo a Eq. (2), obtém-se:

$$
J \ddot{\theta}_{m}+B_{\theta} \dot{\theta}_{m}=T_{m}-\frac{F_{u} p}{2 \pi}
$$

Onde cada variável e parâmetro da Eq. (3) está descrito com sua unidade de medida (no SI) conforme a Tab. 2.

Tabela 2 - Descrição dos parâmetros e variáveis do modelo do eixo-fuso

\begin{tabular}{c|c|c}
\hline Parâmetro/Variável & Descrição & Unidade \\
\hline$J$ & Momento de inércia do eixo motor & $\mathrm{kgm}^{2}$ \\
\hline$\theta_{m}$ & Ângulo de giro do fuso & $\mathrm{Rad}$ \\
\hline$B_{\theta}$ & Coeficiente de atrito viscoso do eixo do motor & $\mathrm{Nms}$ \\
\hline$T_{m}$ & Torque do motor & $\mathrm{Nm}$ \\
\hline$F_{u}$ & Força da reação da massa mola deslocada & $\mathrm{Nm}$ \\
\hline$p$ & Passo do fuso & $\mathrm{m} / \mathrm{rad}$ \\
\hline
\end{tabular}

De modo semelhante, aplicando-se a lei de equilíbrio dinâmico a partir do diagrama de corpo livre da Fig. 3, podem ser deduzidas equações do movimento linear da massa. Assim, considerando o somatório das forças atuantes na massa dado pela Eq. (4), tem-se, na Eq. (5) as forças atuantes.

$$
\begin{gathered}
\sum F=M \ddot{y} \\
F_{u}-B_{y} \dot{y}=M \ddot{y} \\
M \ddot{y}+B_{y} \dot{y}=F_{u}
\end{gathered}
$$

Os parâmetros e variáveis da Eq. (6) estão descritos conforme a Tab. 3, com suas respectivas unidades. 
Tabela 3 - Descrição dos parâmetros e das variáveis do modelo porca-massa

\begin{tabular}{c|c|c}
\hline Parâmetro/Variável & Descrição & Unidade \\
\hline$M$ & Massa deslocada sobre o eixo-fuso & $\mathrm{Kg}$ \\
\hline$y$ & Deslocamento linear da junta prismática & $\mathrm{m}$ \\
\hline$F_{u}$ & Força de reação da massa mola deslocada & $\mathrm{Nm}$ \\
\hline$B_{y}$ & Coeficiente de atrito viscoso da massa & $\mathrm{Nm}$ \\
\hline
\end{tabular}

Se não houvesse a não linearidade da folga no sistema dinâmico, a relação entre o deslocamento linear e o giro do fuso seria descrita através da Eq. (7).

$$
y=\frac{p}{2 \pi} \theta_{m}
$$

Para a existência da folga, se pode fazer um acoplamento cinemático da Eq. (3) e Eq. (6) através da Eq. (8).

$$
y=\left\{\begin{array}{cc}
m\left(\theta_{m}(t)-c_{l}\right) & \text { se } \theta_{m}(t) \leq v_{l} \\
m\left(\theta_{m}(t)-c_{r}\right) & \text { se } \theta_{m}(t) \geq v_{r} \\
y(t-1) & \text { se } v_{l}<\theta_{m}(t)<v_{r}
\end{array}\right.
$$

onde $m, c_{l}(<0)$ e $c_{r}$ são os parâmetros constantes da não linearidade de folga no fuso, e as expressões de $v_{l}$ e $v_{r}$ são dados pela Eq. (9) e pela Eq. (10).

$$
\begin{aligned}
& v_{l}=\frac{y(t-1)}{m}+c_{l} \\
& v_{r}=\frac{y(t-1)}{m}+c_{r}
\end{aligned}
$$

Este modelo será generalizado para os dois graus de liberdade de modo a se chegar a uma equação matricial. Caso o atrito seja expressivo este também será modelado utilizado um sistema de quinta ordem desenvolvido por Ritter (2010).

\section{MODELAGEM DA CINEMÁTICA DIRETA DO ROBÔ}

A modelagem matemática, dentro do contexto da robótica industrial, pode auxiliar os engenheiros pesquisadores na tomada de decisões do projeto mecatrônico e do controle, buscando a otimização do desempenho do sistema, através do estudo e da validação de modelos que descrevem as características geométricas, cinemáticas e dinâmicas (lineares e não lineares). Para este trabalho, buscou-se fazer uso da convenção de Denavit-Hartenberg na modelagem da cinemática direta do robô. Este sistema é descrito em uma matriz de transformação de coordenadas homogêneas da qual resulta a posição e orientação do efetuador final conforme a matriz da Eq. (11) abaixo.

$$
A_{i}^{i-1}=\left[\begin{array}{cccc}
\cos \theta_{i} & -\operatorname{sen} \theta_{i} \cos \alpha_{i} & \operatorname{sen} \theta_{i} \operatorname{sen} \alpha_{i} & a_{i} \cos \theta_{i} \\
\operatorname{sen} \theta_{i} & \cos \theta_{i} \cos \alpha_{i} & -\cos \theta_{i} \operatorname{sen} \alpha_{i} & a_{i} \operatorname{sen} \theta_{i} \\
0 & \operatorname{sen} \alpha_{i} & \cos \alpha_{i} & d_{i} \\
0 & 0 & 0 & 1
\end{array}\right]
$$

A cinemática direta de robôs busca, dados os valores das variáveis de juntas do robô ( $a_{i}$ distância entre os eixos, $Z_{i-1}$ e $Z_{i}, \theta_{i}$ é o ângulo formado entre os eixos $X_{i-1}$ e $X_{i}$ em torno do eixo $Z_{i-1}, d_{i}$ é a ordenada medida ao longo de $Z_{i-1}$ que localiza o eixo $X_{i}$ em relação ao eixo $X_{i-1}$ e $\alpha_{i}$ o ângulo formado entre os eixos $Z_{i-1}$ e $Z_{i}$ medido em torno de $\left.X_{i}\right)$, calcular a posição e a orientação do efetuador final. 


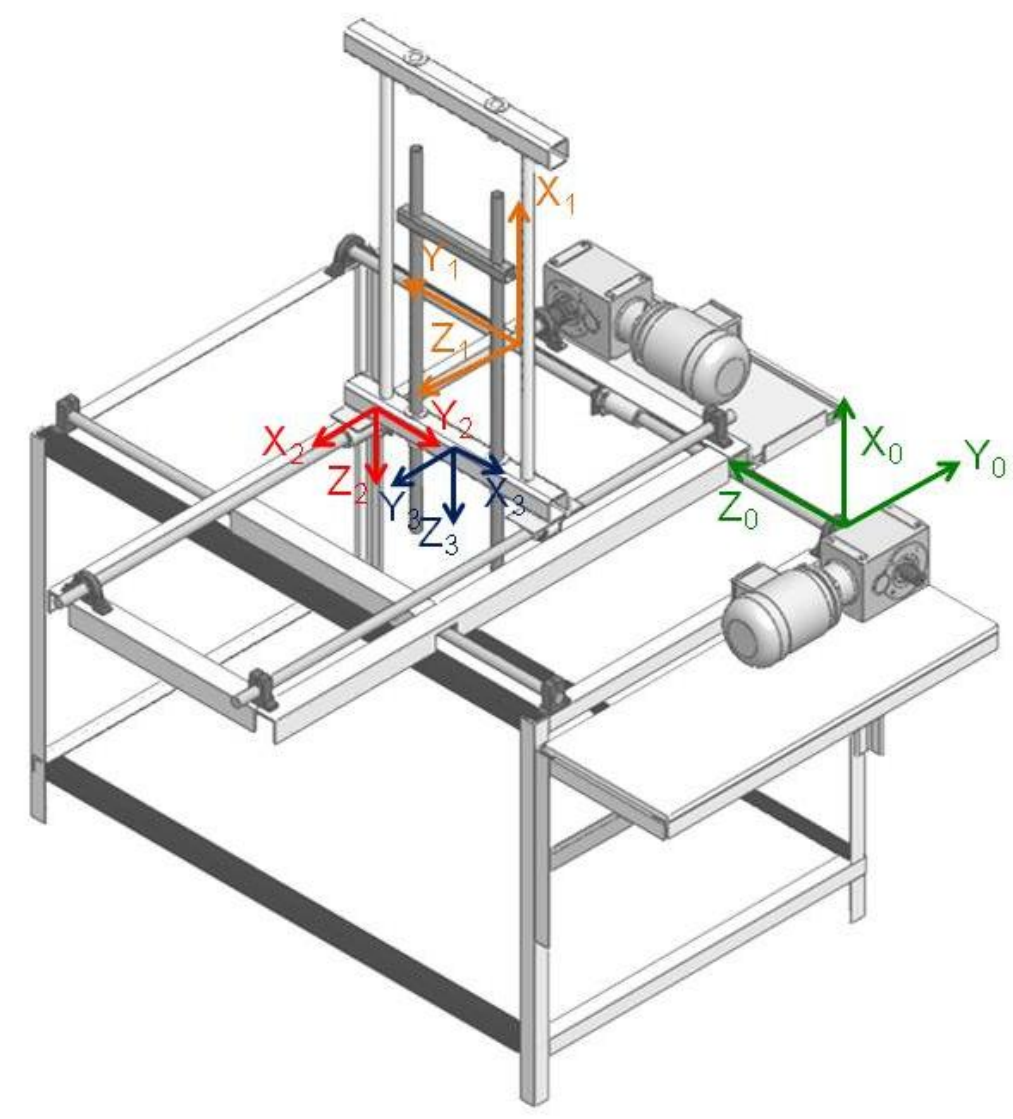

Figura 4 - Eixos coordenados sob cada elo segundo a convenção D-H

Conforme a determinação dos sistemas coordenados de referência em cada elo a partir da convenção D-H indicado na Fig. 4, os parâmetros constam na Tab. 4 abaixo. Os parâmetros constantes serão indicados com suas respectivas referências.

Tabela 4 - Parâmetros de Denavit-Hartenberg

\begin{tabular}{c|c|c|c|c}
\hline elo $_{i}$ & $\boldsymbol{a}_{\boldsymbol{i}}(\boldsymbol{m})$ & $\boldsymbol{\theta}_{\boldsymbol{i}}(\mathbf{r a d})$ & $\boldsymbol{\alpha}_{\boldsymbol{i}}(\mathbf{r a d})$ & $\pi / 2$ \\
\hline $\boldsymbol{e l o}_{\mathbf{1}}$ & $a_{1}$ & 0 & $\pi / 2$ & $d_{1}(\boldsymbol{m})$ \\
\hline $\boldsymbol{e l o}_{2}$ & $a_{2}$ & $-\pi / 2$ & 0 & $d_{2}$ \\
\hline $\boldsymbol{e l o}_{3}$ & 0 & 0 & $d_{3}$ \\
\hline
\end{tabular}

Os parâmetros $a_{3}=\theta_{1}=\theta_{3}=\alpha_{3}=0$, pois os eixos estão sobrepostos e o parâmetro $d_{i}$ é variável. Os parâmetros $a_{1}$ e $a_{2}$ dependem da forma como será construído o robô. Como ainda não se tem o outro grau de liberdade ainda não se pôde fixá-los.

As matrizes de transformação homogênea que relacionam o movimento de um elo em relação ao elo anterior estão descritas através das matrizes das Eq. (12), Eq. (13) e Eq. (14). Substituindo os valores da Tab. 4 na Eq. (11) para cada elo, tem-se:

$$
\begin{aligned}
A_{1}^{0} & =\left[\begin{array}{cccc}
1 & 0 & 0 & a_{1} \\
0 & 0 & -1 & 0 \\
0 & 1 & 0 & d_{1} \\
0 & 0 & 0 & 1
\end{array}\right] \\
A_{2}^{1} & =\left[\begin{array}{cccc}
0 & 0 & -1 & 0 \\
-1 & 0 & 0 & -a_{2} \\
0 & 1 & 0 & d_{2} \\
0 & 0 & 0 & 1
\end{array}\right] \\
A_{3}^{2} & =\left[\begin{array}{llll}
1 & 0 & 0 & 0 \\
0 & 1 & 0 & 0 \\
0 & 0 & 1 & d_{3} \\
0 & 0 & 0 & 1
\end{array}\right]
\end{aligned}
$$


As matrizes acima fornecem a possibilidade de cálculo ponto a ponto da posição, sendo indispensáveis para o planejamento das atividades do manipulador robótico. O produto à esquerda entre Eq. (12), (13) e (14) resulta na matriz de transformação que relaciona o último sistema de referência ao sistema da base conforme Eq. (15).

$$
T_{3}^{0}=\left[\begin{array}{cccc}
0 & 0 & -1 & -d_{3}+a_{1} \\
0 & -1 & 0 & -d_{2} \\
-1 & 0 & 0 & a_{2}+d_{1} \\
0 & 0 & 0 & 1
\end{array}\right]
$$

Seguem abaixo as relações de posição e orientação final do robô. Os vetores das Eq. (16), Eq. (17) e Eq. (18) indicam a orientação do efetuador final em relação à base fixa (elo 0), enquanto o vetor da Eq. (19) indica a orientação da garra ou ferramenta em relação a base fixa.

$$
\begin{gathered}
X_{3}^{0}=\left[\begin{array}{c}
0 \\
0 \\
-1
\end{array}\right] \\
Y_{3}^{0}=\left[\begin{array}{c}
0 \\
-1 \\
0
\end{array}\right] \\
Z_{3}^{0}=\left[\begin{array}{c}
-1 \\
0 \\
0
\end{array}\right] \\
P_{3}^{0}=\left[\begin{array}{l}
P_{x} \\
P_{y} \\
P_{z}
\end{array}\right]=\left[\begin{array}{c}
-d_{3}+a_{1} \\
-d_{2} \\
a_{2}+d_{1}
\end{array}\right]
\end{gathered}
$$

Pode-se, através da variação das variáveis, recalcular as matrizes e se obter novas posições e orientações.

\section{AGRADECIMENTOS}

Aos autores são agradecidos a CAPES pela bolsa de mestrado e à Unijuí pela estrutura laboratorial disponível no Núcleo de Inovação em Máquinas Automáticas e Servo Sistemas (NIMASS) do Câmpus Panambi, implantado com apoio financeiro do FINEP, SEBRAE, CNPq, FAPERGS e MCT.

\section{REFERÊNCIAS}

Douat, L. R.; Queinnec, I.; Garcia, G.; Michelin, M.; Pierrot, F.; Tabouriech, S..2014. Identification and Vibration Attenuation for the Parallel Robot Par2. IEEE Transactions On Control Systems Technology, Vol. 22, No. 1.

Garcia, A. G. P. 2003. Impacto da Lei de Eficiência Energética para Motores Elétricos no Potencial de Conservação de Energia na Indústria. Dissertação de Mestrado (Planejamento Energético - UFRJ). Disponível em: < http://www.ppe.ufrj.br/ppe/production/tesis/agpgarcia.pdf>. Acesso em: 05 jul. 2014. Paatz, S., 2008. Anatomy of a Robot. Engineering \& Technology. p.42-44.

Nof, S. Y. 1999. Handbook of Industrial Robotics. 2 ed. Wiley-Interscience: New York.

Po-Nagen, W. 2009. Real-time Inter-processing for PC Based Robot Controllers in Tele-robotic and Gantry Robotic Controller System. International Conference on Robotics and Biomimetics. Bangkok, Thailand.

Ritter, C. S. 2010. Modelagem Matemática das Características Não Lineares de Atuadores Pneumáticos. Dissertação de Mestrado (Modelagem Matemática - Unijuí).

Romano, V. F. 2002. Robótica Industrial: aplicações na industria de manufatura e processos. São Paulo: Edgar Blücher.

Sciavicco, L.; Siciliano, B. 1996. Modeling and Control of Robot Manipulators. McGraw-Hill: Singapore.

Schilling, R. J. 1990. Fundamentals of Robots Analysis and Control. Englewwod.

Shang, W.; Cong, S., 2014. Motion Control of Parallel Manipulators Using Acceleration Feedback. IEEE Transaction on control, systems technology, vol. 22, n. 1. p. 314-321.

Valdiero, A. C., 2005. Projeto Mecânico de Robôs Industriais.Ijuí: Unijuí.

Linsingen, I. V.. 2003. Fundamentos de sistemas hidráulicos. 2. ed. Florianópolis: UFSC.

Zhang, H.; Zhao, W.; Zhang, J.; Liu, H. 2013. Research on the Modeling of Dynamics for Vertical Axis Ball Screw Feed System. IEEE International Symposium on Assembly and Manufacturing. p. 208-213. 


\title{
RESPONSABILIDADE AUTORAL
}

"O(s) autor(es) é(são) o(s) único(s) responsável(is) pelo conteúdo deste trabalho".

\section{MATHEMATICAL MODELING OF MECHANICAL TRANSMISSION ON A ROBOTIC JOINT WITH BALL-SCREW}

\author{
Angelo Fernando Fiori, an@unochapeco.edu.br ${ }^{1}$ \\ Sandra Baratto Viecelli, sandra_edinara@ hotmail.com $^{2}$ \\ Leonardo Bortolon Maraschin, leonardo.maraschin@ unijui.edu.br ${ }^{3}$ \\ Ismael Barbieri Garlet, Ismael.garlet@ hotmail.com ${ }^{4}$ \\ Antonio Carlos Valdiero, valdiero@ unijui.edu.br ${ }^{5}$ \\ Luiz Antonio Rasia, rasia@ unijui.edu.br ${ }^{6}$ \\ ${ }^{1}$ Regional Northwest University of Rio Grande do Sul - Department of Exact Sciences and Engineering (DCEEng) ... \\ Street Prefeito Rudi Franke, 540, Arco Î́ris - 98280000, Panambi (RS) \\ ${ }^{2}$ Regional Northwest University of Rio Grande do Sul - Department of Exact Sciences and Engineering (DCEEng) ... \\ Street Prefeito Rudi Franke, 540, Arco Íris - 98280000, Panambi (RS) \\ ${ }^{3}$ Regional Northwest University of Rio Grande do Sul - Department of Exact Sciences and Engineering (DCEEng) ... \\ Street Prefeito Rudi Franke, 540, Arco Íris - 98280000, Panambi (RS) \\ ${ }^{4}$ Regional Northwest University of Rio Grande do Sul - Department of Exact Sciences and Engineering (DCEEng) ... \\ Street Prefeito Rudi Franke, 540, Arco Íris - 98280000, Panambi (RS) \\ ${ }^{5}$ Regional Northwest University of Rio Grande do Sul - Department of Exact Sciences and Engineering (DCEEng) ... \\ Street Prefeito Rudi Franke, 540, Arco Íris - 98280000, Panambi (RS) \\ ${ }^{6}$ Regional Northwest University of Rio Grande do Sul - Department of Exact Sciences and Engineering (DCEEng) ... \\ Street Prefeito Rudi Franke, 540, Arco Íris - 98280000, Panambi (RS)
}

\begin{abstract}
The objective is develop the mathematical modeling of the nonlinear characteristics of mechanical transmission in an industrial robot type Gantry and present the design of a prototype. The robot consists of prismatic joints and ball screw driven by electric AC motors. The torque generated at the output of the gearbox results in the angular displacement of the spindle shaft, whose movement is transformed into linear movement of a link on the previous in orthogonal directions, causing him to relocate. The mathematical formulation of the equations of the prismatic joints position becomes an easier task due to kinematic decoupling between the orthogonal joints of the robot. The torque and position data can be obtained from experiments where the sensor signals are captured through an acquisition and control board (dSPACE). However, the mechanical transmissions have nonlinearities that hinder the precision control and impair performance, causing delays and loss of motion. Such as modeling will be in the transmission system geared motor coupling screw-nut, has been the formulation of mathematical models using the Newton-Euler method in dynamic equilibrium from the free-body diagrams of the screw-shaft and nut displaced-mass. Is thus obtained two ordinary differential equations of second order (one for rotation and one for translation). Due to the existence of non-linearity of backlash, these two equations are combined and coupled through a mathematical relationship in discrete time. This dynamic model of prismatic joint is generalized to the two degrees of freedom. As future job prospects, it is planned to experimentally validate the prototype of the robot already in road building. It is hoped that this work will contribute to schemes of compensation for non-linearity and precise control of industrial robots low cost in unhealthy, dangerous and high mechanical stress tasks.
\end{abstract}

Keywords: Mathematical Modeling, Ball-Screw, Non-linearity of Backlash. 